\title{
A Hysteresis Model for an Orthogonal Thin-Film Magnetometer
}

\author{
RENÉ M. DE RIDDER AND JAN H. FLUITMAN, MEMBER, IEEE
}

\begin{abstract}
The operation of a ferromagnetic thin-film magnetometer using the anisotropic magnetoresistance effect in a permalloy film is discussed. Measurements showed the presence of a hysteresis effect that was not predicted by the available models. It is shown that the sensitivity of the magnetometer is correctly predicted by applying Greene and Yarbrough's orthogonal susceptibility model, and that the hysteresis can be explained by assuming dispersion in the magnitude of anisotropy. The ortogonal susceptibility model then has to be evaluated numerically, accounting for a finite driving field. The effect of an inhomogeneous demagnetizing field in the film is discussed in relation to magnitude dispersion of the anisotropy.
\end{abstract}

\section{INTRODUCTION}

$\mathrm{T}$ HE results presented here apply to a class of orthogonal thin-film magnetometers, first reported by West et al. [1], where an alternating excitation field is applied perpendicular to the field to be measured, which is oriented parallel to the easy axis of magnetization of the applied film. Gise and Yarbrough [2] have reported on the influence of tapering the edges of a cylindrical film on the hysteresis of a thin-film magnetometer, without making an attempt to model this phenomenon. No other discussions on the hysteresis occurring in this class of magnetometers to our knowledge have appeared in the literature. In the approach taken here, the ferromagnetic thin film is considered as a continuum, and no attempt has been made to go into micromagnetic detail.

A specific embodiment of the considered class of magnetometers, employing the anisotropic magnetoresistance effect in a planar Hall configuration, has been chosen as the vehicle for this study. It has been shown before [3], [4] that for low operating frequencies and small film dimensions, the magnetoresistance rather than the inductive readout of the magnetic state of the film will, in principle, lead to a better sensitivity. The feasibility of fabricating this type of magnetometer with an integrated drive coil using planar technology has been demonstrated [5]

\section{Magnetometer Operation}

An alternating (e.g., sinusoidal) bias field $H_{\text {ex }}$ is applied perpendicular to the measurand and to the easy axis of a magnetoresistive film which is shaped into a planarHall element or a magnetoresistor having a canted current

Manuscript received April 10, 1989: revised February 20, 1990 The authors are with the Department of Electrical Engineering, Univer sity of Twente, 7500 AE Enschede, The Netherlands.

IEEE Log Number 9035343. path. The bias field periodically saturates the film, and upon returning from saturation, the magnetization splits into two parts (distributed over a number of magnetic domains) which rotate toward the easy axis in opposite directions. The easy-axis field component determines the ratio of the two magnetization fractions, which in turn determines the sensor's output voltage.

In order to explain the operation of the magnetometer in more detail, some models describing the magnetic and galvanic behavior of thin ferromagnetic films will be briefly reviewed.

\section{The Magnetic Behavior of Thin Ferromagnetic Films}

Ferromagnetic phenomena in thin films, in particular, those of permalloy $\left(\mathrm{Ni}_{81} \mathrm{Fe}_{19}\right)$ are treated in many texts, e.g., [6]-[8]. The well-known Stoner-Wohifarth singledomain model gives, in most cases, an adequate description of the behavior of films showing uniaxial anisotropy (like permalloy), and forms a good starting point for developing more complicated models in many other cases.

In permalloy, the axis of anisotropy (easy axis) is in the plane of the film. The orientation perpendicular to the easy axis and in the plane of the film is referred to as the hard axis.

The Stoner-Wohlfarth model leads to an implicit relation between the applied magnetic field vector and the resulting magnetization vector in the film. It is only in a few special cases that explicit relations can be found. Of practical relevance is the case that the magnetic field $\left(H_{T}\right)$ is applied along the hard axis:

$$
\begin{aligned}
\sin (\psi) & =\frac{H_{T}}{H_{k}} & & \text { for }-H_{k} \leq H_{T} \leq H_{k} \\
\psi & =90^{\circ} & & \text { for } H_{T}>H_{k} \\
\psi & =-90^{\circ} & & \text { for } H_{T} \leftarrow H_{k}
\end{aligned}
$$

where $\psi$ is the angle of the magnetization vector $\boldsymbol{M}$ with respect to the easy axis and $H_{k}$ is the anisotropy field.

Often, a graphical tool, the Stoner-Wohlfarth astroid or critical switching curve, is utilized for determining possible stable magnetization orientations for a given applied magnetic field. In practice, the anisotropy in ferromagnetic thin films is not purely uniaxial. Both the orientation $\alpha$ of the easy axis and the strength of the anisotropy field $H_{k}$ may show dispersion (referred to as angular and magnitude dispersion, respectively). In that case, the 
magnetization in the film may split up into a number of domains under certain applied field conditions. Clearly, then, the Stoner-Wohlfarth single-domain model cannot give an adequate description of the magnetic behavior of the film anymore.

An obvious extension is a multiple single-domain model where the film is considered as a collection of noninteracting regions, each having its own easy-axis orientation $\alpha_{i}$ and anisotropy field $H_{k i}$. The distribution of $\left(H_{k i}, \alpha_{i}\right)$ values can be described using a probability density function $f_{k a}\left(H_{k}, \alpha\right)$. The graphic magnetization construction method would require drawing a large number of astroids having different orientations and magnitudes, drawing the applied field vector, constructing possible magnetization vectors in each astroid, and averaging these over all astroids. For all but the simplest cases, this would be a tedious task, which could only be reasonably performed by a computer. This would suggest that not much is to be gained using the graphic astroid method.

A more appropriate graphic method has been proposed by Coren [9] and is illustrated with Fig. 1. In the so-called anisotropy plane, each point represents a specific $\left(H_{k}, \alpha\right)$ combination.

In Fig. 1(b), the average easy axis e.a. ${ }_{0}$ and the average hard axis h.a. ${ }_{0}$ are drawn in the anisotropy plane, and the probability density function $f_{K a}$ is represented as the height above the anisotropy plane. In Fig. 1(c), the anisotropy distribution is drawn in the form of contours of equal $f_{K a}$. As the anisotropy concerned is not of a directional, but an axial type, each anisotropy value is, in fact, represented twice: both in the left and in the right half-plane because ( $\left.H_{k}, \alpha\right)$ is equivalent to $\left(H_{k}, \alpha+\pi\right)$. The same figure can now also be used for representing the magnetization state of the film by shading the "populated" half of the available anisotropy space. The critical switching curve can be written as

$$
H\left(\sin ^{2 / 3}(\gamma-\alpha)+\cos ^{2 / 3}(\gamma-\alpha)\right)^{3 / 2}=H_{k} .
$$

If $H_{k}$ and $\alpha$ are kept constant, the graph defined in the field plane by the relation between $H$ and $\gamma$ is the wellknown astroid; if, however, $H$ and $\gamma$ are kept constant, a curve known as the inverse astroid [Fig. 1(c)] giving the switching effectiveness of $\boldsymbol{H}$ is defined in the anisotropy plane by the relation between $H_{k}$ and $\alpha$.

If the initial state of the film is assumed to be magnetized "to the left"' in Fig. 1(b) and (c), only the anisotropy "hump" in the left half-plane will be populated. When a magnetic field $H$ is applied at an angle $\gamma$ to the average easy axis e.a.0, the corresponding inverse astroid can be drawn. The part of the film having anisotropy values enclosed by the solid-drawn part of the curve will switch to the corresponding region at $\alpha+\pi$.

The magnetic field $\boldsymbol{H}$ applied to the film can be resolved into "longitudinal" and "transversal" components $H_{L}$ and $H_{T}$, respectively, parallel to the average easy and hard axis.

In the orthogonal thin-film magnetometer considered in this paper, an alternating transversal magnetic field hav-

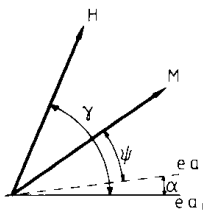

(a)

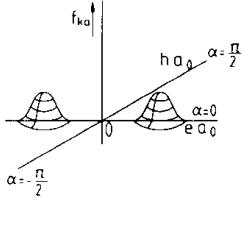

(b)

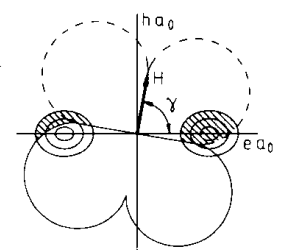

(c)
Fig. 1. Anisotropy space. (a) Definition of angles. (b) Three-dimensional representation of disperse anisotropy. (c) Switching curve (inverse astroid), drawn in the anisotropy plane.

ing an amplitude larger than the maximum $H_{k}$ value is superposed on a relatively weak static longitudinal field $H_{L}$, which is the measurand $\left(H_{L} \ll H_{k}\right)$. When the instantaneous value of $H_{T}$ decreases after having reached a maximum, the magnetization will return to the longitudinal direction. In regions having $\alpha_{i}$ of opposite sign, the magnetization will turn in opposite directions. When $H_{T}$ has decreased to zero, the resulting longitudinal magnetization can have any value from $-M_{s}$ to $+M_{s}$ due to (partial) cancelling out of the contributions from oppositely magnetized regions. The dependence on $H_{L}$ of $M_{L}$ at the moment that $H_{T}=0$, characterized by the orthogonal susceptibility $\chi_{L}$, has been investigated by Green and Yarbrough [10]. The orthogonal susceptibility is defined as

$$
\chi_{L}\left(h_{L}\right)=\left.\frac{\partial m_{L}}{\partial h_{l}}\right|_{h_{T}=0}
$$

where the normalized quantities $h_{L}, h_{T}$, and $m_{L}$ are introduced as

$$
h_{L}=\frac{H_{L}}{H_{k 0}} \quad h_{T}=\frac{H_{T}}{H_{k 0}} \quad m_{l .}=\frac{M_{L}}{M_{s}}
$$

The ferromagnetic film experiences an (applied) field that is the resultant of the longitudinal and transversal field components. The locus of the tip of the resulting field vector is a straight line parallel to and at a distance $H_{L}$ from the transversal axis (Fig. 2(a)). Attached to the field vector is an inverse astroid, contracting and expanding periodically with $H_{T}$. When a quarter cycle of $H_{T}$ (increasing from $H_{T}=0$ to $H_{T}=H_{T m}$ ) is considered, the magnetization in the film regions having anisotropy points enclosed by the envelope of the set of inverse astroids will have switched to the opposite state (the dotted region in Fig. 2(a)). In the anisotropy region where $H_{k}>\left|H_{L}\right|$, the edge of the envelope is parallel to the average easy axis e.a. ${ }_{0}$ at a distance $H_{L}$, sweeping through the anisotropy space. A certain region $i$ characterized by $\left(H_{k i}, \alpha_{i}\right)$ where $\left|H_{L}\right|<H_{k i}<H_{T m}$, can be switched if

$$
H_{k i} \sin \left(\alpha_{i}\right) \leq H_{l}
$$

In Fig. 2(b), $f_{K a}$ has once again been drawn in anisotropy space (now in Cartesian coordinates); the anisotropy region not satisfying (5) has been shaded. The resulting 


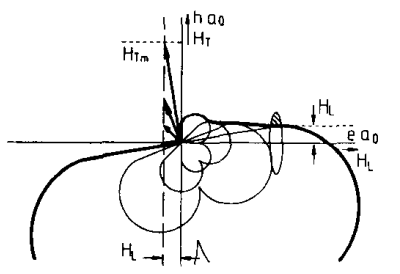

(a)

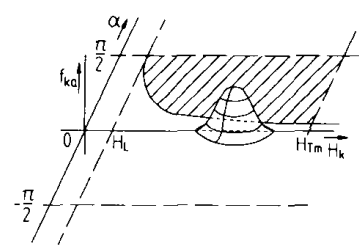

(b)

Fig. 2. (a) Orthogonal drive: inverse astroids and their envelope. switching condition. (b) Integration region for finding the longitudinal magnetization component $M_{l,}$ at the instant that the transversal magnetic-field component $H_{T}=0$

mangnetization can be calculated as

$$
\begin{aligned}
\frac{M_{L}}{M_{s}}= & -1+2 \int_{H_{k}=H_{L}}^{+H_{T m}} \int_{\alpha=\arcsin \left(h_{H .}\right)}^{+(\pi / 2)} f_{K a}\left(H_{k}, \alpha\right) \\
& \cdot \cos (\alpha) d H_{k} d \alpha .
\end{aligned}
$$

In principle, $\chi_{L}$ can be calculated from this expression. A solution in closed form can be found if only angular dispersion is present. Then (6) can be reduced to

$$
m_{L}=2 \int_{\alpha=0}^{\arcsin \left(h_{L}\right)} f_{a}(\alpha) \cos (\alpha) d \alpha .
$$

The orthogonal susceptibility is found by differentiating (7) with respect to $h_{L}$ :

$$
\chi_{L}=2 f_{a}\left(\arcsin \left(h_{L}\right)\right) .
$$

If a normal distribution of angular dispersion is assumed, having a standard deviation $\sigma_{a}$, the orthogonal susceptibility is well approximated by

$$
\chi_{L}=\frac{2}{\sigma_{a} \sqrt{2 \pi}} \exp \left(-\frac{h_{L}^{2}}{2 \sigma_{a}^{2}}\right)
$$

if $\left|h_{L}\right| \ll<$. The result is shown in Fig. 3 where $m_{L}$ has been calculated as

$$
m_{L}=\int_{h_{i}^{\prime}=0}^{h_{L}} \chi_{L}\left(h_{L}^{\prime}\right) d h_{L}^{\prime} .
$$

The linear approximation

$$
m_{L}=\chi_{L O} h_{L}
$$

with the initial orthogonal susceptibility $\chi_{L O}$ given by

$$
\chi_{L O}=\chi_{L}\left(h_{L}=0\right)=\frac{2}{\sigma_{a} \sqrt{2 \pi}}
$$

is valid for small values of $h_{L}$ and causes less than $1 \%$ error in $m_{L}$ if $\left|h_{L}\right|<0.25 \sigma_{a}$.

If both angular and magnitude dispersion are present in a film, a simple closed form like (9) cannot be obtained, but Greene and Yarbrough [10] have shown that for a normally distributed magnitude dispersion, having a sufficiently small standard deviation $\sigma_{K}$, the initial orthogonal susceptibility has the same value as in the case without

(a)

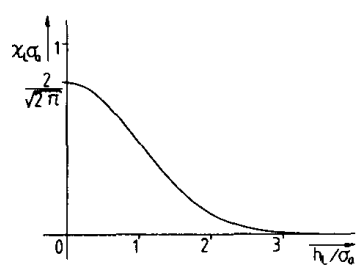

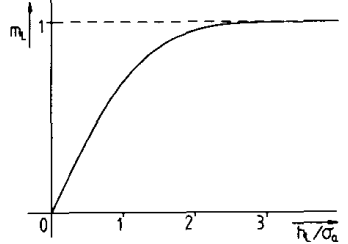

(b)
Fig. 3. (a) Orthogonal susceptibility and (b) normalized magnetization for a normal distribution of $f_{t}$ and no magnitude dispersion of $H_{k}$ versus normalized longitudinal magnetic-field component.

magnitude dispersion:

$$
\chi_{L O}=\frac{2}{\sigma_{a} \sqrt{2 \pi}} \quad \text { for } \sigma_{K} \ll H_{k 0} / 5
$$

\section{The Anisotropic Magnetoresistance Effect}

The phenomenology of galvanomagnetic effects is well known (e.g., [11]). One of these effects, the anisotropic magnetoresistance effect, is characterized by the ratio $\Delta \rho / \rho_{0}$ where

$$
\Delta \rho=\rho_{\|}-\rho_{\perp} \text { and } \rho_{0}=\left(\rho_{\|}+\rho_{\perp}\right) / 2 .
$$

The physical phenomena, giving rise to the anisotropic resistivity coefficients $\rho_{\|}$and $\rho_{\perp}$, which themselves are not dependent on the magnetic field or magnetization, have been described by McGuire and Potter [12]. The resistance anisotropy, linked to the magnetization vector in the material, may give rise to an electrical field component perpendicular to the direction of current flow (pseudo- or planar Hall effect). The conventional Hall configuration is shown in Fig. 4(a). If disturbing geometrical effects due to e.g., the short-circuiting effect of the current contacts on the planar Hall field are neglected, the planar Hall voltage is given by

$$
V_{H}=\frac{1}{2} j w \Delta \rho \sin (2 \varphi)
$$

where $j$ is the current density in the film, $w$ is the width of the film, $\Delta \rho$ is the absolute resistance anisotropy as defined in (14), and $\varphi$ is the angle of the magnetization vector $\boldsymbol{M}$ with respect to the current density vector $\boldsymbol{j}$. In the case of a transversal applied field, (1) and (15) can be combined to read

$$
\begin{array}{ll}
V_{H}= \pm j w \Delta \rho h_{T} \sqrt{1-h_{T}^{2}} & \text { for }\left|h_{T}\right| \leq 1 \\
V_{H}=0 & \text { for }\left|h_{T}\right|>1
\end{array}
$$

where the + sign applies if the magnetization is in the first and fourth quadrants and the - sign in the remaining quadrants. $V_{H}$ versus $H_{T}$ is shown in Fig. 4(b).

If anisotropy dispersion cannot be neglected, the magnetization in the film will be inhomogeneous in the orthogonal drive configuration. The most simple model would consider only two domains, having their common domain wall parallel to the easy axis (and to $j$ ), where the sense of rotation of $\boldsymbol{M}$ is opposite for both domains. The 


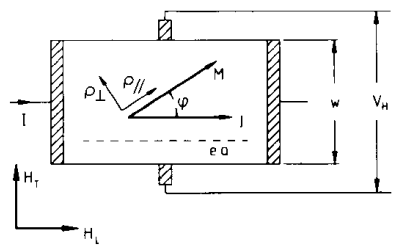

(a)

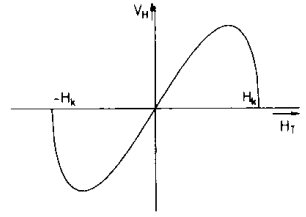

(b)
Fig. 4. (a) Geometry of a thin-film device showing the anisotropic magnetoresistance effect. (b) Planar Hall effect.

proportion of the widths of both domains depends on the longitudinal field and can be calculated using the orthogonal susceptibility model (10). It is assumed that the position of the domain wall is not directly affected by $h_{T}$, and that both domains independently obey the StonerWohlfarth model. If anisotropy dispersion is small, which is the case for good quality permalloy films, and $\left|h_{L}\right|$ $<1$, the domains will contribute to the planar Hall voltage according to (16) with positive and negative sign, respectively, leading to

$$
\begin{gathered}
V_{y}=j w \Delta \rho h_{T} \sqrt{1-h_{T}^{2}} \int_{h i=0}^{h_{l}} \chi_{L}\left(h_{L}^{\prime}\right) d h_{L}^{\prime} \\
\text { for }\left|h_{T}\right|<1 \\
V_{y}=0 \text { for }\left|h_{T}\right| \geq 1
\end{gathered}
$$

or, using (11) in the linear approximation (for $\left|h_{L}\right| \ll$ $\left.\sigma_{a}\right)$,

$$
\begin{array}{ll}
V_{y}=j w \Delta \rho \chi_{L O} h_{L} h_{T} \sqrt{1-h_{T}^{2}} & \text { for }\left|h_{T}\right|<1 \\
V_{y}=0 & \text { for }\left|h_{T}\right| \geq 1 .
\end{array}
$$

If $H_{T}$ is an alternating field, the planar Hall voltage will traverse curves as in Fig. 4(b), with an amplitude reduced by a factor $\chi_{L O} h_{L}$. In particular, the Hall voltage will be zero if $h_{L}=0$.

\section{Magnetometer Model}

Using the models summarized above and neglecting dispersion in the magnitude of anisotropy and shape effects, the output voltage for the planar Hall configuration can be written as

$$
V_{H}=V_{0} S\left(H_{s}\right) E\left(H_{\mathrm{ex}}\right)
$$

where $H_{s}$ has been chosen parallel to the easy axis, and $V_{0}$ defines the basic galvanomagnetic sensitivity as

$$
V_{0}=\frac{1}{2} j w \Delta \rho
$$

with $j$ the current density, $w$ the width of the film, and $\Delta \rho$ the absolute resistance anisotropy.
$S\left(H_{s}\right)$ gives the primary sensitivity to the measurand, based only on parameters of the orthogonal sensitivity model:

$$
\begin{aligned}
S\left(H_{s}\right)= & \frac{2}{H_{k} \sigma_{a} \sqrt{2 \pi}} \int_{H_{1}^{\prime}=0}^{H_{u}} \\
& \cdot \exp \left[-\frac{1}{2}\left(\frac{H_{s}^{\prime}}{H_{k} \sigma_{a}}\right)^{2}\right] d H_{s}^{\prime}
\end{aligned}
$$

with $H_{k}$ the anisotropy field and $\sigma_{a}$ the standard deviation of angular dispersion of anisotropy. $E\left(H_{\mathrm{ex}}\right)$ is the (timedependent) excitation factor

$$
\begin{array}{ll}
E\left(H_{\mathrm{ex}}\right)=2 \frac{H_{\mathrm{cx}}}{H_{k}} \sqrt{1-\left(\frac{H_{\mathrm{ex}}}{H_{k}}\right)^{2}} & \text { for }\left|H_{\mathrm{ex}}\right|<H_{k} . \\
E\left(H_{\mathrm{ex}}\right)=0 & \text { for }\left|H_{\mathrm{cx}}\right| \geq H_{k}
\end{array}
$$

The basic functional shape of $S\left(H_{s}\right)$ is equal to that of $m_{L}\left(h_{L}\right)$ (Fig. 3(b)), and the shape of $E\left(H_{\mathrm{ex}}\right)$ corresponds to $V_{y}\left(h_{T}\right)$ in Fig. $4(\mathrm{~b})$.

As $H_{\mathrm{cx}}$ is an alternating field having an amplitude larger than $H_{k}$, then $V_{H}$ will be an alternating signal having a peak-to-peak value $V_{s i:}$ :

$$
V_{s s}=2 V_{0} S\left(H_{s}\right) \text {. }
$$

If $H_{s}$ is not oriented parallel to the easy axis, it can be resolved into components $H_{L}$ and $H_{T}$, respectively parallel and perpendicular to this axis. The perpendicular component $H_{T}$ is parallel to $H_{\mathrm{ex}}$. If the amplitude of the driving field $\hat{H}_{\text {ex }}$ is large enough to ensure periodical saturation of the film (in which case $\hat{H}_{\mathrm{ex}} \gg H_{k}+\left|H_{s}\right|$ ), $V_{s s}$ will be independent of $H_{T}$. Of course, the $E\left(H_{\mathrm{ex}}\right)$ function defined in (22) will change. At least the values of $H_{\mathrm{ex}}$ for which it reaches an extremum will change, but the extreme values themselves will remain unchanged, so that $V_{s s}$ is determined only by $H_{L}$. Therefore, in principle, a true vector component can be measured, and if the measurand $\boldsymbol{H}_{s}$ is applied at an angle $\boldsymbol{\varphi}$ to the easy axis, (23) should be modified to read

$$
V_{s}=2 V_{0} S\left(\left|\boldsymbol{H}_{s}\right| \cos (\varphi)\right) .
$$

For very low applied fields $\left(\left|\boldsymbol{H}_{s}\right| \ll H_{k} \sigma_{a}\right)$, the exponential factor in $(21)$ is equal to unity, so in that case, the first-order approximation

$$
S\left(H_{s}\right)=S_{0} H_{s}=\frac{2}{H_{k} \sigma_{a} \sqrt{2 \pi}} H_{s}
$$

is justified.

\section{Measurements}

Measurements have been performed on $10 \times 10 \mathrm{~mm}^{2}$ permalloy films having a thickness of about $50 \mathrm{~nm}$. These large films have been chosen for reducing shape anisotropy to a level which can be neglected with respect to intrinsic anisotropy. The films were contacted using con- 
ducting rubber strips in a conventional Hall configuration as shown in Fig. 4(a).

The peak-to-peak output planar Hall voltage $V_{s s}$ versus $H_{s}$ has been measured for various amplitudes of the sinusoidally alternating drive field $H_{\mathrm{ex}}$, in which $H_{s}$ is the magnetic-field component perpendicular to the drive field. The rate of change of $H_{s}$ has been chosen low with respect to the frequency of $H_{\mathrm{ex}}$, so that $H_{s}$ can be considered constant during one period of $H_{\mathrm{ex}}$.

Some typical results are shown in Figs. 5-7.

Fig. 5(a) shows the output voltage $V_{H}$ versus time during one period of $H_{\mathrm{ex}}$ when $H_{s}$ has a small value. In the region near the zero crossing of $H_{\mathrm{ex}}$ (where $H_{\mathrm{cx}}$ varies approximately linearly with time), the similarity of $V_{H}$ to Fig. 4(b) (described by (16)) can be seen. For large values of $H_{\text {ex }}$, the film is approximately saturated in the hard direction, resulting in zero output voltage. Fig. 5(b) depicts the same signal $V_{H}$ versus $H_{\mathrm{ex}}$, showing the planar Hall effect even more obviously. If $H_{s}$ is zero (Fig. 5(c)), $V_{H}$ should be identically zero for all values of $H_{\mathrm{ex}}$. It can be seen that in reality, some noisy residual signal remains.

In Fig. 6(a), the peak-to-peak value $V_{s s}$ of $V_{H}$ is shown versus $H_{s}$. The values are normalized with respect to $2 V_{0}$ (20) and $H_{k} \sigma_{a}$, respectively. $H_{k}$ has been measured using Kneer's modified planar Hall method [13], yielding a value of $H_{k}=320 \mathrm{~A} / \mathrm{m}$. The angular dispersion of anisotropy was found to be $\sigma_{a}=1.1^{\circ}$, using Crowther's type I method [14]. Keeping the value of $H_{k}$ at $320 \mathrm{~A} / \mathrm{m}$, a best fit of the measurement results to the calculations using (19) was obtained with $\sigma_{a}=1.0^{\circ}$. The $10 \%$ discrepancy is within the accumulated measurement error.

Minor deviations from the predicted curve occur for higher fields, the approach to saturation being slower than predicted. The same deviation is not found in the Crowther-type measurements. Crowther [14] showed in the discussion on his alternative measurement method II that this type of deviation can be expected if angular and magnitude dispersion have correlated distributions where high $H_{k}$ values correlate with high angular deviations.

The results of these measurements show that the orthogonal susceptibility model can quite accurately predict the sensitivity (especially in the low-field region) of the orthogonal thin-film magnetometer under discussion. Equation (21) gives an adequate description of the functional dependence of $V_{s s}$ on $H_{s}$, and the linear approximation (25) is valid for calculating the low-field sensitivity up to fields of about $0.4 \sigma_{a} H_{k}$.

The amplitude of the driving field does not influence the sensitivity. It should be noted, however, that the shape of $V_{H}$ versus time (such as shown in Fig. 5(a)) does change with driving-field amplitude, as a larger driving field causes the film to be saturated for a larger part of the excitation period, and consequently causes the output pulses to become narrower. If the signal processing equipment is based on an average value measurement, this reduced duty cycle may lead to decreased overall sensitivity.

The linear part of the curve around the origin is magnified and shown in Fig. 6(b). A hysteresis phenomenon

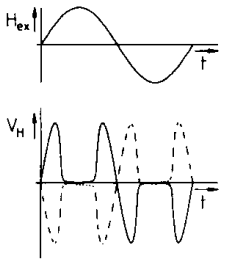

(a)

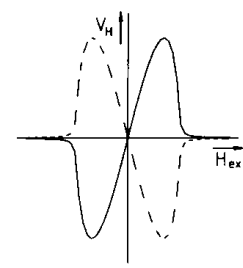

(b)

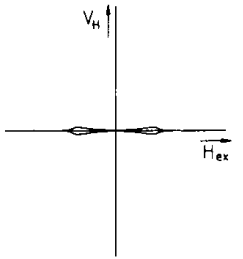

(c)
Fig. 5. Magnetometer operation. (a) $H_{c x}$ and $V_{H}$ versus time. (b). (c) $V_{t t}$ versus $H_{\mathrm{cx}}$ ((a) and (b): solid curve: $H_{\mathrm{s}}=H_{s 0} \neq 0$, dashed curve: $H_{s}=$ $-H_{s i}$; (c) $H_{s}=0$ ).

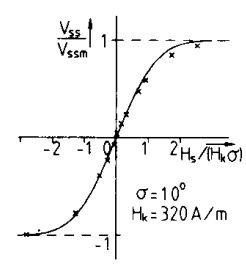

(a)

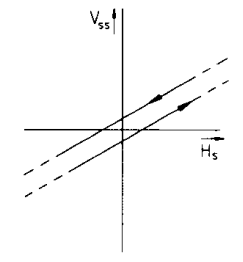

(b)

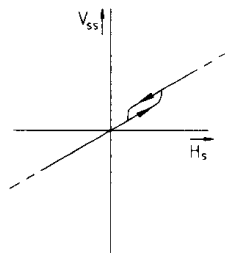

(c)
Fig. 6. (a) Normalized peak-to-peak Hall voltage $V_{w} /\left(2 V_{t}\right)$ versus normalized easy-axis field $H_{s} /\left(H_{k} \sigma_{t}\right)$. Positive and negative values are related to the phase of $V_{H}$ with respect to $H_{\mathrm{ex}}$ (cf. solid and dashed curves in Fig. 5). Solid line is calculated; crosses denote measured values. (b), (c) Hysteresis phenomena.
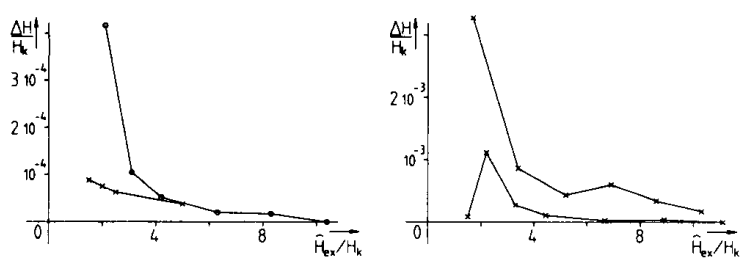

Fig. 7. Hysteresis versus driving field amplitude for various films.

is found, which is unexpected at first. Fig. $6(\mathrm{c})$ shows a rather "local" hysteresis phenomenon which could be observed in only a few samples.

In order to find out the origin of this hysteresis, the field uncertainty at the origin $\Delta H$ in Fig. 6 has been measured versus the amplitude of the excitation field. The results are shown in Fig. 7.

\section{HYSTERESIS}

The hysteresis in the magnetometer response as shown in Fig. 6(b) and (c) is somewhat surprising at first sight. After all, the film is assumed to be completely saturated every half cycle by the excitation field, so any remnant of previous magnetic states should be erased. Several causes of hysteresis are conceivable.

- External:

- immediate proximity of ferromagnetic materials, experiencing and influencing the measurand $H_{s}$;

- measurements performed under unsuitable conditions (changing $H_{s}$ too fast with respect to the dominant time constant of the measurement and registration equipment). 
- Internal:

- The film is not saturated at all, due to the finite value of the driving field and

- magnitude dispersion of the anisotropy field;

- inhomogeneity of the internal field in the film due to the demagnetizing field.

After eliminating the external causes (part of the hysteresis proved to be caused by the presence of a nickel interlayer in gold-plated electrical contacts), some hysteresis still remained. First, a qualitative argument will be presented to show that both internal causes mentioned can produce a memory effect and thus hysteresis; next, a more quantitative assessment of the hysteresis will be made using the available models.

\section{A Simple Two-Region Hysteresis Model}

Consider a film, consisting of two regions 1 and 2, having different anisotropy fields $H_{k 1}$ and $H_{k 2}$, but for simplicity, equal orientations of the easy axes. In Fig. 8, the critical switching curves (concentric astroids) of both regions have been partially drawn. Assuming that the driving field has an amplitude $\hat{H}_{\mathrm{ex}}$ with $H_{k 1}<\hat{H}_{\mathrm{ex}}<H_{k 2}$, and that the measurand $H_{s}$ varies slowly with respect to $H_{\mathrm{ex}}$, then the trajectories described by the terminal point of the resultant magnetic-field vector will be straight lines parallel to the (common) hard axis and at a distance $H_{s}$ from this axis (vertical dashed lines in Fig. 8). Only one stable magnetization direction exists if the terminal point of the magnetic-field vector is outside the astroid, and two magnetization directions are possible if the vector is inside the astroid. In region 1 , the vector tip will be outside the switching curve at the peak values of $H_{\mathrm{ex}}$ for any value of $H_{s}$. This is not necessarily the case for the other region. If, for example, $H_{s}=H_{s 1}$, only one magnetization state $\boldsymbol{M}_{1}$ is possible at the peak of $H_{\mathrm{ex}}$ in region 1, but two states $\boldsymbol{M}_{2}$ and $\boldsymbol{M}_{3}$ are possible in region 2, depending on the history of $\boldsymbol{M}$ in this region (e.g., the state will be $\boldsymbol{M}_{2}$ if the previous value of $H_{s}$ was $H_{s 2}$, but it will be $M_{3}$ if $H_{s 3}$ was the previous measurand value). Different starting states like $\boldsymbol{M}_{2}$ and $\boldsymbol{M}_{3}$, and consequently different series of "connected" states occurring during a cycle of $H_{\mathrm{cx}}$ will, in general, lead to contributions of opposite sign to the galvanomagnetic output voltage. The resulting dependence of the output voltage on both the present value and history of the measurand explains the hysteresis effect.

It should be expected that the hysteresis will be reduced as the driving field amplitude is increased because then a larger part of the regions of the film will be saturated, leaving less space for ambiguities.

This is observed as a general trend (Fig. 7(a)), but exceptions are found showing local minima and maxima (Fig. 7(b)).

These exceptions may be explained by assuming the existence of discrete high-anisotropy regions which do not switch (and so do not cause hysteresis) for low driving fields, switch partially (causing hysteresis) for intermediate driving fields, and are saturated (again no hysteresis) for high driving fields.

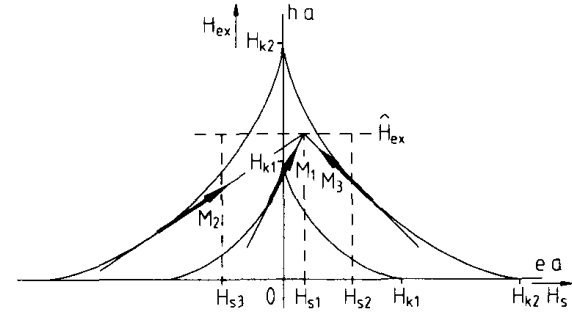

Fig. 8. Simple model for explaining hysteresis: film consisting of two regions having different $H_{k}$, but equal easy-axis orientation: driving field with peak value $\hat{H}_{\mathrm{cx}}$ along the hard axis, measurand $H_{s}$ along the easy axis.

\section{An Extended Orthogonal-Susceptibility Model}

If more quantitative statements about the hysteresis phenomenon are required, a model is needed. The simple two-region model used above is insufficient for describing the behavior of real films.

The orthogonal susceptibility model, as it has been used here, cannot account for hysteresis because (9) and thus (19) have been derived under the restriction of zero magnitude dispersion or limited magnitude dispersion and infinite driving field, respectively.

If the infinite-excitation field condition which was necessary in Greene and Yarbrough's treatment [10] is dropped, the model can predict hysteresis, as will be illustrated referring to Fig. 9.

For various applied fields $H_{s}(n)$ and $H_{\mathrm{ex}}(m)$, stationary states $S_{n m}$ and intermediate states $I_{n m}$ have been symbolically depicted (in the anisotropy plane) in the diagrams of Fig. 9. Three specific values of the driving field $H_{\text {ex }}$ have been chosen for each value of the measurand $H_{s}$ : the maximum positive $(m=a)$ and negative $(m=b)$ values and zero $(m=c)$. The resultant field vectors and relevant parts of the associated inverse astroids have been drawn. The horizontal line segments are parts of the envelope of the family of inverse astroids associated with the particular value of $H_{s}$ (cf. Fig. 2(a)). The dotted lines in some of the diagrams indicate parts of the relevant inverse astroids associated with previous states (the history of the material). For the sake of clarity and ease of drawing, the anisotropy distribution is assumed to have a sharp rectangular boundary, but this is not an essential restriction. The populated part of the anisotropy space has been shaded. The double-headed arrows indicate possible transitions from one state to another.

The intermediate states can only exist for, at most, a half cycle of the driving field. In the case of a constant value $H_{s}(n)$, the two states $S_{n a}$ and $S_{n b}$ alternate. The $S_{n c}$ states depict the situation where the maximum longitudinal magnetization component is detected. In principle, two such states should be distinguished: either returning from positive or from negative excitation. However, the two states are mirror images of each other with respect to the horizontal (longitudinal) axis, leading to equal longitudinal magnetization components. Returning from positive excitation, the regions $C_{1}$, and in the opposite case, the 

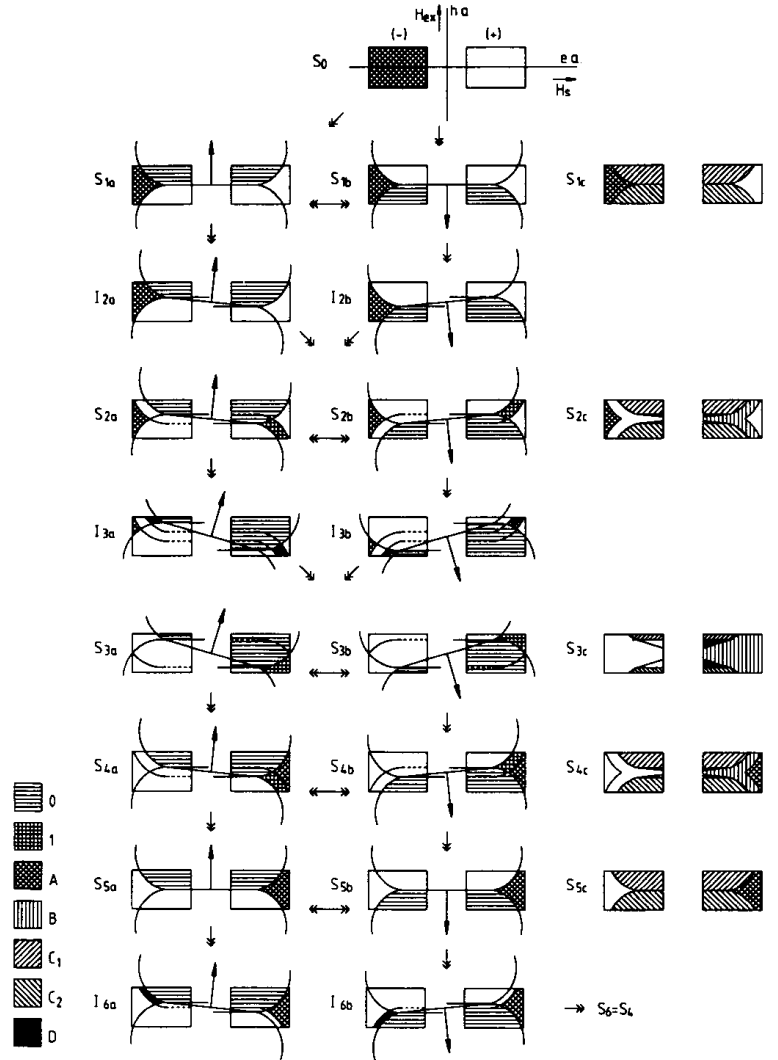

Fig. 9. Hysteresis explained using the extended orthogonal susceptibility model. All diagrams have been drawn in anisotropy space. $S_{i}$ : stationary states, $I_{i}$ : intermediate states, column a: $H_{\mathrm{cx}}=+\hat{H}_{\mathrm{ux}}$, b: $H_{\mathrm{cx}}=-\hat{H}_{\mathrm{cx}}$, c: $H_{\mathrm{ex}}=0$. Shaded regions are "populated": type of shading: $\mathrm{O}$ : switched by presently valid envelope of inverse astroids, 1 : inherited from another state for the same value of $H_{v}$. A: inherited from another state for a previous value of $H_{3}$, B: determined completely by $H_{3}$ (static), $C_{1}, C_{2}$ : populated only after respectively positive and negative excitation cycles, D: dynamically switched each cycle by "sweeping action" of inverse astroid envelope. (A more detailed explanation of the diagrams is given in the main text.)

regions $C_{2}$, are populated. This gives rise to remanence in the transversal direction, which is not relevant for this discussion.

A starting state $S_{0}$, magnetized "to the left," is assumed. This orientation of magnetization is defined as the negative direction. An alternating driving field is now applied keeping $H_{s}=0$, which leads to states $S_{1 a}$ and $S_{1 b}$ where part of the magnetization (determined by the inverse astroid) is periodically switched by the alternating field, and the remaining part (cross shaded) is not at all affected by the field. The state of this last part has been inherited from the previous state (in this case, $S_{0}$ ).

If a small constant field $H_{s}$ is superposed on the alternating driving field, initially $S_{1 a}$ or $S_{1 b}$ transform to $I_{2 a}$ or $I_{2 b}$, respectively, which in turn, on reversal of $H_{\mathrm{cx}}$, transform to stationary states $S_{2 b}$ or $S_{2 a}$, respectively.

Four different regions of magnetization $A-D$ can be distinguished in diagram $S_{2 c}$ : region $A$ (negative) is not af- fected by the field and is inherited from the previous state; $B$ (positive) is completely determined by the applied field, but is not periodically switched; $C$ (either $C_{1}$ or $C_{2}$, depending on the excitation phase) (positive and negative) is periodically switched by the field in such a way that positive and negative contributions always cancel each other out exactly; $D$ (positive and negative) is also periodically switched, but in such a way that, for near-zero $H_{\mathrm{ex}}$, the resulting contribution is always positive.

The field $H_{s}$ is so large in state $S_{3}$ that region $A$ is reduced to zero, and the state is completely determined by the present magnetic field, i.e., the hysteresis disappears. When $H_{s}$ is again decreased, the film will occupy state $S_{4}$, which corresponds to $S_{2}$ (equal $H_{2}$ values). It can be seen that region $A$ has changed sign, leading to a higher value of $M_{L}$, and $S_{5}$ shows the state when $H_{s}$ is again reduced to zero. Region $A$, which is the only nonzero magnetization region in this case, has changed sign with respect to $S_{1}$. If from this state $H_{s}$ is again increased, traversing $I_{6}$, a state $S_{6}$ will be reached which is identical to $S_{4}$.

In Fig. 10(a), the longitudinal magnetization component at $H_{\mathrm{ex}}=0$ has been drawn versus $H_{s}$. Thus, one half of a hysteresis curve is constructed by connecting the states $S_{1}-S_{6}$ discussed above.

If a negative $H_{s}$ is applied, the diagrams of Fig. 9 will be mirrored with respect to the transversal (vertical) axis, and the rest of the hysteresis curve will be found by mirroring with respect to the origin.

This graphical method could be continued for constructing minor hysteresis loops (e.g., by reducing $H_{s}$ in $\left.S_{2}\right)$.

The maximum field uncertainty $\Delta H_{s}$ can be calculated from $\Delta M_{L}$, using the linear approximation of the hysteresis curve around the origin (Fig. 10(b)) with (11):

$$
\Delta H_{s}=\frac{\Delta M_{L} H_{k 0}}{M_{s} \chi_{L O}}=\frac{\Delta M_{L}}{M_{s}} \frac{1}{S_{0}} .
$$

Of course, $\Delta H_{s}$ is independent of the method for determining $\Delta M_{L}$ (e.g., inductively or using the planar Hall effect).

The maximum uncertainty in $M_{L} / M_{s}$, which occurs for $H_{s}=0$, is equal to twice the magnetization region indicated by $A$ in Fig. 9, and it is calculated by integrating the probability density function of anisotropy over the region outside both inverse astroids associated with the positive and negative extreme values of $H_{\mathrm{ex}}$.

This is illustrated in Fig. 11.

$$
\frac{\Delta M_{L}}{M_{s}}=4 \int_{\alpha=0}^{\pi / 2}\left\{\int_{H_{k}=H_{b}}^{\infty} f_{K a}\left(H_{k}, \alpha\right) d H_{k}\right\} d \alpha
$$

where the boundary $H_{b}\left(\alpha, H_{s}, \hat{H}_{\mathrm{ex}}\right)$ is given by the inverse astroid (2), which can be written as

$$
\begin{aligned}
H_{b}= & \left\{\left\{\hat{H}_{\mathrm{ex}} \cos (\alpha)+H_{s} \sin (\alpha)\right\}^{2 / 3}\right. \\
& \left.+\left\{H_{s} \cos (\alpha)-\hat{H}_{\mathrm{ex}} \sin (\alpha)\right\}^{2 / 3}\right\}^{3 / 2} .
\end{aligned}
$$

Expression (27) has been calculated numerically for several different parameters. Independent angular and mag- 


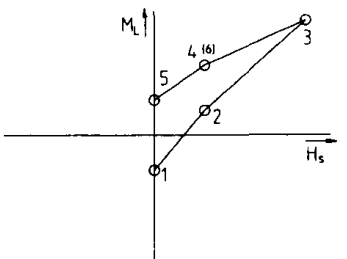

(a)

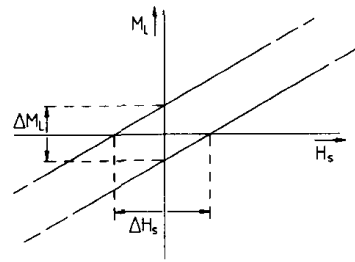

(b)
Fig. 10. Hysteresis curves. (a) Constructed from Fig. 9. (b) Linear approximation of hysteresis curve for small $H_{\text {. }}$.

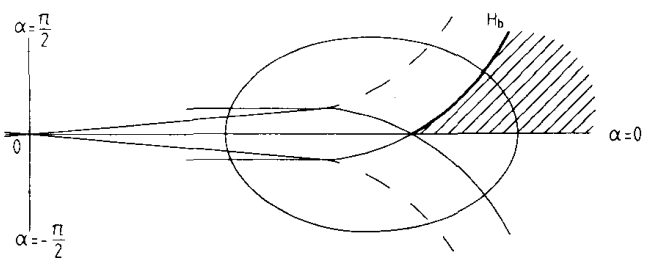

Fig. 11. Integration region for calculating maximum hysteresis (27).

nitude dispersion distributions have been assumed so that $f_{K a}\left(H_{k}, \alpha\right)=f_{K}\left(H_{k}\right) f_{a}(\alpha)$. The angular distribution has been taken as normal, having a standard deviation $\sigma_{a}=$ $1^{\circ}$. Two types of distribution have been tried for the magnitude dispersion: a normal and an exponential one. For a normal $H_{k}$ distribution having a standard deviation $\sigma_{k}=$ $0.1 H_{k 0}$, Fig. 12 shows the calculated hysteresis versus $\hat{H}_{\text {ex }} / H_{k 0}$ for three different values of $H_{s} / H_{k 0}$. In Fig. 12(a), the ratio $\Delta M_{L} / M_{s}$ is given over the full hysteresis range. Of course, for normal operation of the magnetometer, only the section of the curves for $\hat{H}_{\mathrm{ex}} / \mathrm{H}_{k} \mathrm{O}>1$ is relevant. Fig. 12(b) shows the maximum hysteresis expressed in (normalized) field units, calculated using (26); here, only the low range of hysteresis is displayed ( for a normal permalloy film having $H_{k 0}=350 \mathrm{~A} / \mathrm{m}$, full scale corresponds to $0.035 \mathrm{~A} / \mathrm{m}$ ).

In Fig. 13, similar diagrams are shown for a film having an exponential magnitude probability density function:

$$
\begin{gathered}
f_{K}\left(H_{k}\right)=\frac{1}{\sigma_{k}} \exp \left(-\frac{H_{k}-H_{k 0}+\sigma_{k}}{\sigma_{k}}\right) \\
\quad \text { for } H_{k} \geq H_{k 0}-\sigma_{k} \\
f_{K}\left(H_{k}\right)=0
\end{gathered}
$$

$$
\text { for } H_{k}<H_{k 0}-\sigma_{k}
$$

with $\sigma_{k}=0.1 H_{k 0}$.

This distribution has a longer tail than the normal distribution, which is reflected in the hysteresis curves (note that the $\hat{H}_{\mathrm{ex}} / H_{k 0}$ scale in Fig. 13(b) has been shifted with respect to this scale in Fig. 12(b)). Crowther [14] already indicated that real $H_{k}$ distributions are not symmetrical, but must have a high-field tail. Evidence for the existence

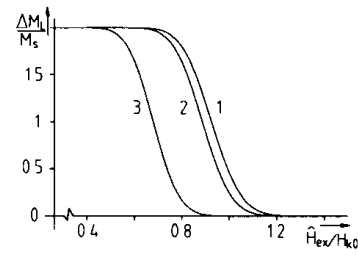

(a)

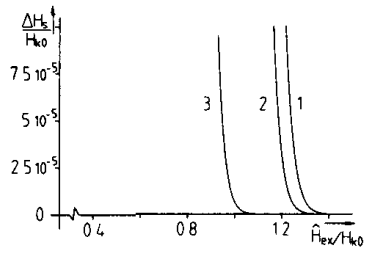

(b)
Fig. 12. Calculated hysteresis of a magnetometer employing a permalloy film having independent normal distributions of angular and magnitude dispersion with $\sigma_{a}=1^{\circ}, \sigma_{k}=0.1 H_{k(1)}$. Curves: $1: H_{s}=0,2: H_{s}=$ $0.01 H_{k(1} .3: H_{,}=0.1 H_{k 0}$. Diagrams: (a) magnetization uncertainty, (b) field uncertainty versus normalized amplitude of excitation field.

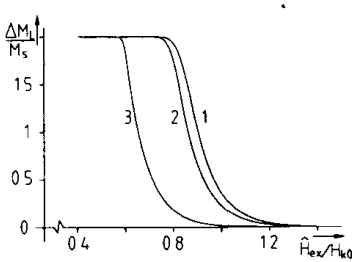

(a)

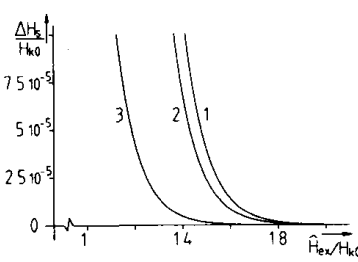

(b)
Fig. 13. Calculated hysteresis curves obtained with an exponential $H_{k}$ distribution having $\sigma_{k}=0.1 H_{k \mid l}$. The other conditions are identical to those in Fig. 12.

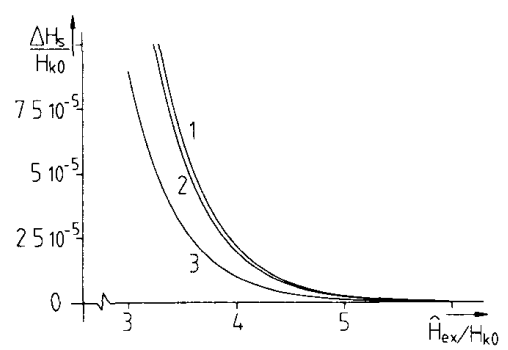

Fig. 14. Calculated field uncertainty for a film with an exponential $H_{k}$ distribution having $\sigma_{k}=0.5 \mathrm{H}_{k 0}$.

of these tails has also been found in systematic deviations from theoretical behavior of ordinary magnetoresistive devices [15].

Finally, in Fig. 14, the field uncertainty is given for a film having an exponential $H_{k}$ distribution with $\sigma_{k}=$ $0.5 H_{k 0}$. It is clear that in this case, a rather large amplitude of the excitation field is necessary for reducing hysteresis to a low level.

\section{Discussion}

These calculations show that hysteresis can be caused by dispersion in the magnitude of anisotropy. In order to prove that magnitude dispersion is the cause, this dispersion and the correlation coefficient with angular dispersion should be measured. However, this has not yet been done because a reliable measurement was not available.

More complicated anisotropy distributions, showing two or more different maxima, would be necessary for 
explaining a "local" hysteresis phenomenon like that shown in Fig. 6(c).

Furthermore, hysteresis measurements appeared to be dependent on measurement conditions. A possible explanation is the occurrence of stress-induced anisotropy where a stress variation is caused by the method of mounting and contacting the devices. A similar effect has been observed in noise measurements.

It has been shown by Eijkel [16] that our films do exhibit some residual magnetostriction indeed.

Although the $H_{k}$ distributions used are somewhat arbitrary, the trend of the calculated hysteresis curves is confirmed by measurement (Fig. 7).

In the models presented above, magnitude dispersion of the anisotropy has been assumed. In films having a large width, the average demagnetizing field may be much smaller than the anisotropy field $H_{k}$, so that one would expect the effects of demagnetization to be negligible with respect to the intrinsic anisotropy. In general, however, this demagnetizing field will be very inhomogeneous. In the case of a rectangular edge profile, a field strength equal to $M_{s}(800 \mathrm{kA} / \mathrm{m}$ for permalloy) is required for completely saturating the film. It has been shown that tapering the film edges significantly reduces the field required for complete saturation.

In the case of an inhomogeneous demagnetizing field, two regions having identical astroids will experience different magnetic-field vectors. This situation can be scaled to the case shown in Fig. 8, and then the same reasoning will apply.

In practice, the difference between the two cases can be quite vague; for example, microscopic defects (inhomogeneities, pinholes, scratches, cracks) in the film could possibly cause strong local inhomogeneities in the demagnetizing field. On the other hand, due to their stochastic character, the resulting inhomogeneous distribution of magnetization can be attributed to anisotropy dispersion. Indeed, dispersion of anisotropy is only a model which is sometimes useful for describing the magnetic behavior of an anisotropic film, and it does not necessarily correspond to a single real physical phenomenon.

It has been indicated that the inhomogeneous internal field due to demagnetization has an effect on hysteresis similar to the one magnitude dispersion has. It should be noted, however, that it is not correct to describe the demagnetization effect as magnitude dispersion as was done by Kwiatkowski et al. [17], although this seems to be an attractive approach from a computational point of view. This "dispersion" will be field-dependent as soon as the film becomes partially saturated.

\section{Conclusions}

The operation of the orthogonal thin-film magnetometer using the anisotropic magnetoresistance effect can be largely explained by a model based on Greene and Yarbrough's orthogonal susceptibility model. For low fields, the sensitivity is correctly predicted (within experimental error) by the model. The linear approximation of sensitivity is valid for fields up to approximately $0.4 \sigma_{u} H_{k}$.

Hysteresis of the device can be explained by the effects of dispersion in the magnitude of anisotropy and of the inhomogeneous demagnetizing field. A modified orthogonal susceptibility model can be used for calculating hysteresis if the distribution of the anisotropy dispersion is given.

\section{REFERENCES}

111 F G. West W. J Odom, J A. Rice, and T. C. Penn, "Detection of low-intensity magnetic fields by means of ferromagnetic films." $J$ Apply: Phys. vol. 34. pp. 1163-1164. 1963.

21 P. E. Gise and R. B. Yarbrough. “An improved cylindrical magnetometer sensor." IEEE Trans. Magn., vol. MAG-13, pp. 1104-1106. 1977.

13) R. M. de Ridder and J. H. Fluitman. "Orthogonal thin-film magne tometer using the anisotropic magnetoresistance effect, " IEEE Trams. Magn., vol. MAG-20, pp. 690-692, 1984

[4] R. M. de Ridder. "Thin-film magnetoresistive magnetometer. "Ph.D dissertation. Univ. Twente. The Netherlands, 1988.

[5] R. Groothengel and R. M. de Ridder, “Thin-film magnetometer with integrated planar drive coil." in Proc. 3rd Sensors Actuators Simp. J. C. Lodder, Ed. Kluwer, 1986, pp. 211-218.

[6] S. Middelhoek, Ferromagnetic Domains in Thin NiFe Films. Amsterdam: van Soest, 1961.

17] R. F. Soohoo. Magnetic Thin Films. New York: Harper \& Row, 1965

[8] M. S. Cohen. "Ferromagnetism in films." in K. L. Chopra, Thin Film Phenomena. New York: MeGraw-Hill. 1969. pp. 608-720.

[9] R. L. Coren. "A description of magnetic switching in disperse an isotropy films," J. Appl. Phys., vol. 35. pp. 201-205. 1964

[10] F. S. Greene and R. B. Yarbrough, "Orthogonal susceptibility of permalloy films," J. Appl. Phys., vol. 41. pp. 4076-4082. 1970.

111] J.-P. Jan. "Galvanomagnetic and thermomagnetic effects in metals," Solid State Physics, vol. 5. F. Seitz and D. Turnbull. Eds. New York: Academic, 1957, pp. 1-96.

[12] T. R. McGuire and R. I. Potter. "Anisotropic magnetoresistance in ferromagnetic $3 \mathrm{~d}$ alloys," IEEE Trans. Magn., vol. MAG-11. pp. $1018-1038.1975$.

[13] G. Kneer, "The anisotropy field Hk of thin ferromagnetic films, measured by magnetoresistive methods," IEEE Trans. Magn.. vol. MAG-2. pp. 747-750. 1966.

[14] T. S. Crowther. "Angular and magnitude dispersion of the anisotropy in magnetic films. "J. Appl. Phis.. vol. 34. pp. 580-587. 1963.

[15] R. M. de Ridder and J. H. J. Fluitman, "Modelling the (galvano-) magnetic behavior of permalloy sensors." J. de Physique. vol. 46 pp. C6-287-C6-290. 1985

116] K. J. Eijkel. "A thin-film magnetoresistive angle detector." Ph.D. dissertation, Univ. Twente. The Netherlands. 1988

[17] W. Kwiatkowski, M. Stabrowski, and S. Tumanski, "Numerical analysis of the shape anisotropy and anisotropy dispersion in thin film permalloy magnetoresistors, "IEEE Trans. Magn., vol. MAG-19, pp. $2501-2505.1983$.

René M. de Ridder was born in Amsterdam. The Netherlands, on April 26, 1950. He studied electrical engineering at the University of Twente. Enschede, The Netherlands, where he received the Master's degree in 1978 and the Ph.D. degree in 1988.

Since 1981 he has been employed as a Lecturer and Research Scientist at the University of Twente.

Jan H. Fluitman (M 80 ) was born in Beverwijk, The Netherlands, on March 23, 1938. He received the M.Sc. and Ph.D. degrees in physics from the University of Amsterdam in 1966 and 1970, respectively.

He joined the University of Twente, Enschede. The Netherlands, in 1970. teaching applied magnetism and conducting research in the field of magnetic recording and magnetic transducers. Since 1982 he has been a Full Professor of Transducer Science. His interests now are in magnetic. optic, and micromechanic transduction principles. 